\title{
25 Research Square \\ Computational analysis on Covid-19 transmission escalates into new imuuno-epidemiology considerations.
}

Anthony Kyriakopoulos ( $\square$ antkyriak@gmail.com )

Nasco AD Biotechnology Laboratory

\section{Shi Zhao}

JC School of Public Health and Primary Care, Chinese University of Hong Kong, Hong Kong 999077, China Jinhui Li

JC School of Public Health and Primary Care, Chinese University of Hong Kong, Hong Kong 999077, China

\section{Research Article}

Keywords: SARS-COV-2 dispersion, Heterogeneity, Individual infectiveness, Age groups, Transmission dynamics.

Posted Date: February 5th, 2021

DOI: https://doi.org/10.21203/rs.3.rs-199986/v1

License: (c) (i) This work is licensed under a Creative Commons Attribution 4.0 International License. Read Full License 


\section{Abstract}

\section{Background}

For Severe Acute Respiratory Syndrome Coronavirus-2, the investigation of the heterogeneity of individual infectiousness is important due to the recorded widespread cross reactive immunity of general population that can alter transmission dynamics. We therefore aimed to understand how SARS-COV-2 transmits in the general population in relation to age.

\section{Design}

Using a sample of infected population with SARS-COV-2 in close geographical proximity to the initial Severe Advanced Respiratory Syndrome-1 (SARS-1) outbreak, we explored the association between infector's age and dispersion (or heterogeneity) of individual infectiousness $(k)$ in order to investigate the relatedness with the age of an individual's capability to disperse SARS-COV-2.

\section{Results}

We have found a negative association between $\mathrm{k}$ and increase of infector's age. Significantly this becomes more evident for the age group of 20-60 years comparing with the infectors of younger age.

\section{Conclusions}

Non pharmaceutical interventions can be effective to age group between 20-60 years whereas in youngsters and older patients containment of spreading must be made by other means to be effective. Immunity differences between age groups may reflect their differences in heterogeneity predicted by variance in dispersion parameter (k).

\section{Introduction}

The resistance of the "host-donor" defines the efficient transmission of the infectious agents [1]. This resistance can be described as the immunity of individuals raised to disable transmission [1-2]. The previous Severe Acute Respiratory Syndrome-1 (SARS-1) patients, even after 17 years post infection poses a sustained-well-developed, specific T cell memory response against SARS Coronavirus-1 (SARS COV-1). This specific immunity is intensified according to the increased severity of previous SARS-1 clinical condition [2]. These SARS-1 patients whilst all poses a long term cross reactive immunity against SARS-1-COV, also all (to a tested sample of 23), poses reacting T cells to the $\mathrm{N}$ peptides of SARS-COV-2 [3]. Further, the unexposed individuals with no previous history of SARS-1 infection, and negative for Nucleocaspid protein ( $\mathrm{N}$ ) antibodies and neutralizing antibodies of SARS-COV-2, to a $51.35 \%$ (19/37), have also a reactive T cell immunity against SARS-COV-2 N, and non structural proteins (nsp) proteins of SARS-COV-2 (50\% and $50 \%$ respectively) [3]. Also a widely distributed T cell cross reactivity (35\%$60 \%$ ) amongst unexposed individuals extends against to the SARS-COV-2 spike (S) protein [4-5].

The specific memory immunity of unexposed individuals is attributed either to immune cross reactions with common flu coronavirus infections [4-5] or to the involvement of animal species cross transmitting coronaviruses to human [3]. Since there is a 17 year interval from SARS-1 epidemic and the developmental stages of immunity can be severely influenced [6-7] by the cross barrier transmission of coronaviruses (including common cold 
coronaviruses) between animal species and human individuals (as shown in figure 1), we have selected specifically to investigate the variation of dispersion parameter $k$ with age increase, bearing in mind the concept of higher $k$ à less heterogeneity of age group à more difficult to control the epidemic [8]. We considered that investigation on this route could identify distinct variation in individual infectiveness with the new virus and thus identify variations on the efficiency of individuals to spread SARS-COV-2 between specific age groups.

Figure. 1. Cross species barrier transmission between human and animals by coronaviruses. The cross species barrier infection is achieved by coronaviruses using suitable receptors that enable this transmission. Molecules like the carcinoembryonic antigen-related adhesion molecules (CEACAM) comprise a family of antigens which are highly preserved between animal and human species and their participation can lead to a wide immune cellular dispersal of coronaviruses throughout the human organism [9].

\section{Patients And Methods}

\section{Data collection and extraction}

We used the Coronavitus Disease-19 (COVID-19) surveillance data previously published [20] and the dataset can be accessed freely via the public respiratory https://github.com/linwangidd/covid19_transmissionPairs_China/blob/master/transmission_pairs_covid_v2.csv.

The dataset contains 1407 transmission pairs that are identified and reconstructed according to the previous studies [8, 21], governmental new release, and official situation reports.

We identified 807 infectors, and who act as source of infection to transmit to the infectee. We extract the information, including age and gender, of each infector as well as the number of offspring infectees generated by each infector. After excluding the infector with missing information on age, we collected 777 infectors for further analysis.

\section{Heterogeneity of individual infectiousness: a statistical modeling perspective}

We consider the variation in the individual-level infectiousness as a quantifiable scale that affects the distribution of offspring infectee generated by an infector. Following the previous study [21], we introduce the number of offspring infectee generated by an infector, denoted by $r$, as a random variable from a Gamma distribution, denoted by $h($ ), with mean $R(>0)$ and dispersion parameter $k(>0)$. Thus, we have $r \sim h(R, k)$. Here, $R$ is the reproduction number that is defined as the expected (or average) number of secondary cases caused by one typical infected individual.

The dispersion parameter $k$ governs the dispersiveness of the Gamma distribution. As demonstrated theoretically in the previous study [21], with $R$ fixed, a larger $k$ results in a lower effectiveness of non-pharmaceutical interventions in controlling the epidemics, which is also discussed in another study [8].

Poisson process with rate $r$, denoted by $f(r)$, is adopted to address the stochastic effects in transmission, and to govern the number of infectee caused by each infector, denoted by $Z(\geq 0)$. [22]. Thus, we have $Z \sim f(r)=f(R, k)$. 
Straightforwardly, $f(R, k)$ is an Negative Binomial (NB) distribution with mean $R$ and variance $R \bullet(1+R / k)$. By the definition of NB distribution, the probability that one infector generates $(j-1)$ offspring infectees, i.e., cluster size of $j(\geq 1)$, which is denoted by $\operatorname{Pr}(Z=j-1)=L_{j}$, is given in Eqn (1).

$$
\operatorname{Pr}(Z=j-1)=L_{j}=\frac{\Gamma(k j+j-1)}{\Gamma(k j) \cdot \Gamma(j+1)} \cdot \frac{\left(\frac{R}{k}\right)^{j-1}}{\left(1+\frac{R}{k}\right)^{k j+j-1}}
$$

Here, $\Gamma(\bullet)$ denotes the Gamma function. Specially, the NB distribution $f(\bullet)$ reduces to a Geometric distribution when $k=1$, and it reduces to a Poisson distribution when $k$ approaches infinity. Importantly, a smaller value of $k$ indicates larger heterogeneity in individual infectiousness.

By fitting distribution $f(\bullet)$ to the real-world observations, we may estimate value of dispersion parameter $k$, and explore the determinants of $k$.

\section{Likelihood inference framework and subgrouping by infector's age}

We considered observed samples of number of offsprings from Ninfectors. We denoted the number of infectors who have $j$ infectees associated by $n_{j}(\geq 1)$. Note that all $j>1$ in our dataset though $j$ may be 1 theoretically or observed in other datasets, and thus we adjusted for this truncation in our likelihood framework. Straightforwardly, we have. Then, following the previous studies [23, 24], we constructed the likelihood function, denoted by $L$, as in Eqn (2).

$$
L=\prod_{j>1}\left(\frac{L_{j}}{1-L_{1}}\right)^{n_{j}}
$$

We estimated the dispersion parameter $k$ using the maximal likelihood estimation approach. To explore the association between the infector's age and $k$, we repeated the above fitting and estimation procedure after subsetting the dataset into subgroups by the infector's age. We considered 76 age bins, and they include 5-25, 6-26, ..., 79-99, and $80+$ years. We estimate the value of $k$ for each age bin to examine the association between the infector's age and $k$.

\section{Results}

We estimated the $k$ ranges from 0.4 to 1.5 for different age bins, which is line with previous studies [8, 23, 25]. We observe an evident downward trend in $k$ as the infector's age increases, with $p$-value $<0.001$ for linear trends testing using Student's $t$ test. We detect a structural break [26] at age bin 20-40 years, in which $k$ drops $47 \%$ with $p$ value $<0.001$ comparing with the infector with younger age, presented in figure 2 .

\section{Discussion}

We have employed the metric named dispersion of individual infectiousness, denoted by $k$, which was first proposed for SARS-1 [21] because it was used to quantify the role of heterogeneity of individuals in transmitting Covid-19 as well as the difficulty in controlling the epidemics with Non Pharmaceutical Interventions (NPIs) at population scale $[23,25]$. We performed a statistical calculation of $k$, and explored to define possible relatedness 
of infector's age as a determinant of $k$. We estimated the $k$ ranges from 0.4 to 1.5 for different age bins in line with the previous studies $[8,23,25]$. We observe an evident downward trend in $k$ as the infector's age increases, with $p$ value $<0.001$ for linear trends testing using Student's $t$ test. Moreover, we detect a structural break at age bin 2040 years, in which $\mathrm{k}$ drops $47 \%$ with $\mathrm{p}$-value < 0.001 comparing with the infector with younger age (see figure 2 ). The dataset [see [20] for full list of transmission pairs] we used to investigate variation in heterogeity of individual infectiveness with age initially contained 1407 transmission pairs [25], out of which we have identified 807 infectors. Out of these infectors, we selected 777 pairs with adequate with information about age.

In the investigation of individual infectiveness we kept the reproduction number $\mathrm{R}$ constant in order to measure the variation of individual dispersiveness, $k$, with age. The $k$ value decreases with age increase, with the important difference being between the two age groups $0-20$, and $20-60$ years of age (see figure 2 ). This is important, as their difference in $k$ value lies in the heterogeneity of each sub-population group [8,21]. For youth age as $k$ is larger, the heterogeneity of subgroup population is smaller and for the older age group as $k$, decreases the heterogeity between individual increases $[21,25,27]$. As presented in figure 3 , when hypothesizing that $R$ equals to 2 , which is a realistic scenario for SARS-COV-2 [28], for four seed cases of youth and old age respectively, the offspring cases will be eight for each age group. This means that almost all youth age infectors will produce two offspring cases, whereas for old age infectors, almost half will not be able to produce any offspring case, one will be able to produce six offspring cases and one two offspring cases.

Reflecting the change of individual infectiveness with age and thus their heterogeneity, NPIs, are more applicable to the sub-population older than 20 years of age [21], whereas NPIs are not expected to provide an adequate solution [21, 29], for disease spread containment from younger age infectors. Given the situation that a high proportion of youth age remains asymptomatic but highly infectious [6, 30], this makes the contact tracing effort in this group even more difficult but urgent. Specific screening strategy for the youth population to identify as more possible positivity will make restriction contact measures more efficient as almost all young infected individuals are likely to transmit the disease. The notable differences in the heterogeity of individuals across the age groups of 0-20, 20-60 and slightly over 60-90 years old may reflect de-similarities between developments of immune surveillance mechanisms due to environmental cross transmission reactions [10-19]. These are encountered in previous SARS-1 patients and unexposed individuals [3-5]. Due to Covid-19 overspread and consistence, the targeted pharmaceutical interventions may be appropriate to lower seed cases in population groups with small heterogeneity. As our results show, by focusing on youth age population to prevent from spreading SARS-COV-2 to rest of general population, this will help to contain pandemic in a similar way to SARS1.

\section{Conclusions}

NPI's are expected to work efficiently for population between ages of 20-60 years old as with SARS-1. However, this seems not to be the case for younger population infecting SARS-COV-2. For this population other measures are needed to contain infection to general population. For older than 60 years of age sub-population the heterogeneity of infectiousness also decreases but not in the same way as for youngsters. The differences of dispersion ability between age groups can reflect differences in memory immunity acquired post the SARS-COV-1 outbreak as time interval matches. Special cross reactive immunity studies to detect memory immunity in the young population group are needed. Similar testing of dispersion parameter $(k)$ in European and USA are needed to discover trends of hererogeneity across age groups. 


\section{Abbreviations And Acronyms}

NPI: Non Pharmaceutical Intervensions

SARS-1: Severe Acute Respiratory Syndrome-1

SARS-COV-1: Severe Acute Respiratory Syndrome Coronavirus-1

SARS-COV-2: Severe Acute Respiratory Syndrome Coronavirus-2

$\mathbf{N}$ : Nucleocaspid

NSP: Non structural proteins

S: Spike

COVID-19: Coronavirus Disease -19

h: Gamma distribution

$\boldsymbol{R}$ : Reproduction number

$\boldsymbol{k}$. Dispersion parameter

Z: Number of infectees

NB: Negative Binomial

$\Gamma(\cdot)$ : Gamma function

CEACAM: Carcinoembryonic antigen-related cell adhesion molecule

SSE: Super Spread Event

MHV: Mouse Hepatitis Virus

MERS: Middle East Respiratory Syndrome

CoV: Coronavirus

MERS-COV: Middle East Respiratory Syndrome Coronavirus

ACE-2: Angiotensin I Converting Enzyme 2

ARDS: Acute Respiratory Distress Syndrome

DPP-4: Dipeptidyl Peptidase-4

GPI: Glycophosphatidylinositol

ITAM: Immunoreceptor Tyrosine Based Motif 
TGEV: Transmissible Gastroenteritis Virus

IgSF: immunoglobulin superfamily

hAPN: human aminopeptidase N

pAPN: Piglet aminopeptidase N receptor

\section{Declarations}

Ethical approval: Not applicable

Consent for publication: Not Applicable

Availability of data and materials: All literature data are available upon request by the corresponding author, $\mathrm{Dr}$ Anthony M. Kyriakopoulos FIBMS

Competing interests: All authors declare no competing interests.

Funding: This study was funded by The Key Project of Medicine Discipline of Guangzhou (2017-2019-07).

Author contributions: AK had the conception of the study, and SZ materialized the design of mathematical investigation. AK with that aid of $\mathrm{JL}$ analyzed the data, and AK interpreted the immune-epidemiology relevance. AK did the drafting of the paper and $J L$ and SZ revised it critically for intellectual content. All authors agreed to the final version to be published, and all authors agree to be accountable for all aspects of work.

Acknowledgments: We thank Professor J. Kountouras M.D., Ph.D., and Professor Markus Nagl M.D. for their valuable communication on Covid-19 epidemiology and reviewing of manuscript. We thank our families for moral support in conducting this research.

\section{References}

1.Stephenson JF, Young KA, Fox J, et al. Host heterogeneity affects both parasite transmission to and fitness on subsequent hosts. Philos Trans R Soc Lond Biol Sci.2017;372(1719):20160093. DOI: 10.1098/rstb.2016.0093

2. Ng OW, Chia A, Tan AT, et al. Memory T cell responses targeting the SARS coronavirus persist up to 11 years post-infection. Vaccine. 2016; 12;34(17):2008-14. DOI: 10.1016/j.vaccine.2016.02.063.

3. Le Bert N, Tan AT, Kunasegaran K, et al. SARS-CoV-2-specific T cell immunity in cases of COVID-19 and SARS, and uninfected controls. Nature. 2020; 584(7821):457-462. DOI: 10.1038/s41586-020-2550-z.

4. Grifoni A, Weiskopf D, Ramirez SI, et al. Targets of T Cell Responses to SARS-CoV-2 Coronavirus in Humans with COVID-19 Disease and Unexposed Individuals. Cell. 2020; 25;181(7):1489-1501.e15. DOI:

10.1016/j.cell.2020.05.015.

5. Pia L. SARS-CoV-2-reactive T cells in patients and healthy donors. Nat Rev Immunol. 2020; 20(6):353. DOI: $10.1038 /$ s41577-020-0333-2. 
6. Zimmermann P, Curtis N. Coronavirus Infections in Children Including COVID-19: An Overview of the Epidemiology, Clinical Features, Diagnosis, Treatment and Prevention Options in Children. Pediatr Infect Dis. 2020;39(5):355-368. DOI:10.1097/INF.0000000000002660

7. Mesel-Lemoine $\mathrm{M}$, Millet $\mathrm{J}$, Vidalain PO, et al. A human coronavirus responsible for the common cold massively kills dendritic cells but not monocytes. J Virol. 2012;86(14):7577-7587. DOI:10.1128/JVI.00269-12

8. He D, Zhao S, Xu X, et al. Low dispersion in the infectiousness of COVID-19 cases implies difficulty in control. BMC Public Health. 2020;20(1):1558. DOI: 10.1186/s12889-020-09624-2.

9. Rizeq B, Zakaria Z, Ouhtit A. Towards understanding the mechanisms of actions of carcinoembryonic antigenrelated cell adhesion molecule 6 in cancer progression. Cancer Sci. 2018;109(1):33-42. DOI:10.1111/cas.13437

10. Taguchi F, Matsuyama S, Soluble Receptor Potentiates Receptor-Independent Infection by Murine Coronavirus. J Virol. 2002;76(3):950-8.

11.Baric RS, Sullivan E, Hensley L, et al. Persistent infection promotes cross-species transmissibility of mouse hepatitis virus. J Virol. 1999;73(1): 638-649.

12. Wentworth DE, Holmes KV. Molecular determinants of species specificity in the coronavirus receptor aminopeptidase N (CD13): influence of N-linked glycosylation. J Virol. 2001;75(20):9741-9752.

DOI:10.1128/JVI.75.20.9741-9752.2001

13. Hansen $\mathrm{GH}$, Delmas B, Besnardeau L, et al., The coronavirus transmissible gastroenteritis virus causes infection after receptor-mediated endocytosis and acid-dependent fusion with an intracellular compartment. J Virol. 1998;72(1):527-534.

14. Rossen JW, Bekker CP, Voorhout WF, et al. Entry and release of transmissible gastroenteritis coronavirus are restricted to apical surfaces of polarized epithelial cells. J Virol. 1994;68(12):7966-7973.

15. Chan CM, Chu H, Wang Y, et al. Carcinoembryonic Antigen-Related Cell Adhesion Molecule 5 Is an Important Surface Attachment Factor That Facilitates Entry of Middle East Respiratory Syndrome Coronavirus. J Virol. 2016;90(20): 9114-9127. DOI:10.1128/JVI.01133-16

16.Jaume M, Yip MS, Chung Y, et al. Anti-severe acute respiratory syndrome coronavirus spike antibodies trigger infection of human immune cells via a $\mathrm{pH}$ - and cysteine protease-independent FcyR pathway. J Virol. 2011;85(20):10582-10597. DOI:10.1128/JVI.00671-11

17.Kammerer R, Zimmermann W, Coevolution of activating and inhibitory receptors within mammalian carcinoembryonic antigen families. BMC Biol. 2010; 8:12. DOI:10.1186/1741-7007-8-12

18. Booth JW, Telio D, Liao EH, et al. Phosphatidylinositol 3-kinases in carcinoembryonic antigen-related cellular adhesion molecule-mediated internalization of Neisseria gonorrhoeae. J Biol Chem. 2003; 278(16):14037-14045. DOI:10.1074/jbc.M211879200

19. Spiegel M, Schneider K, Weber F, et al. Interaction of severe acute respiratory syndrome-associated coronavirus with dendritic cells. J Gen Virol. 2006; 87(Pt 7):1953-1960. DOI:10.1099/vir.0.81624-0 
20. Xu XK, Liu XF, Wu W, et al., Reconstruction of Transmission Pairs for novel Coronavirus Disease 2019 (COVID19) in mainland China: Estimation of Super-spreading Events, Serial Interval, and Hazard of Infection. Clin Infect Dis. 2020; [published ahead of print 2020 Jun 18:ciaa790. DOI: 10.1093/cid/ciaa790].

21. Lloyd-Smith JO, Schreiber SJ, Kopp PE, et al. Superspreading and the effect of individual variation on disease emergence. Nature. 2005; 17:438(7066):355-9.

DOI: $10.1038 /$ nature04153.

22. Diekmann O, Heesterbeek JAP. Mathematical Epidemiology of Infectious Diseases: Model Building, Analysis, and Interpretation. S. Levin, Ed., Wiley series in mathematical and computational biology (John Wiley \& sons, New York, 2000).

23. Adam DC, Wu P, Wong J, et al. Clustering and superspreading potential of SARS-CoV-2 infections in Hong Kong. Nat Med. 2020; [published ahead of print https://doi.org/10.1038/s41591-020-1092-0]

24. Kucharski AJ, Althaus CL. The role of superspreading in Middle East respiratory syndrome coronavirus (MERSCoV) transmission. Euro Surveill. 2015; 20(25):14-8. DOI: 10.2807/1560-7917.es2015.20.25.21167

25. Kwok KO, Chan HHH, Huang Y, et al. Inferring super-spreading from transmission clusters of COVID-19 in Hong Kong, Japan, and Singapore. J Hosp Infect. 2020; 105(4):682-685. DOI: 10.1016/j.jhin.2020.05.027. 32446721.

26. https://en.wikipedia.org/wiki/Structural_break

27. Woolhouse ME, Dye C, Etard JF, et al., Heterogeneities in the transmission of infectious agents: implications for the design of control programs. Proc Natl Acad Sci U S A. 1997; 94:338-42.

28. Kyriakopoulos AM, A. Papaefthymiou A, N. Georgilas N, et al. The Potential Role of Super Spread Events in SARS-COV-2 Pandemic; a Narrative Review. Arch Acad Emerg Med.2020; 8(1):e74.

29. Riley S, Fraser C, Donelly CA, et al. Transmission dynamics of the etiological agent of SARS in Hong Kong: impact of public health interventions. Science. 2003; 300(5627): 1961-6. DOI:10.1126/science.1086478

30. Oliveira DS, Medeiros NI, Gomes JAS. Immune response in COVID-19: What do we currently know? Microb Pathog. 2020;148:104484.

\section{Figures}




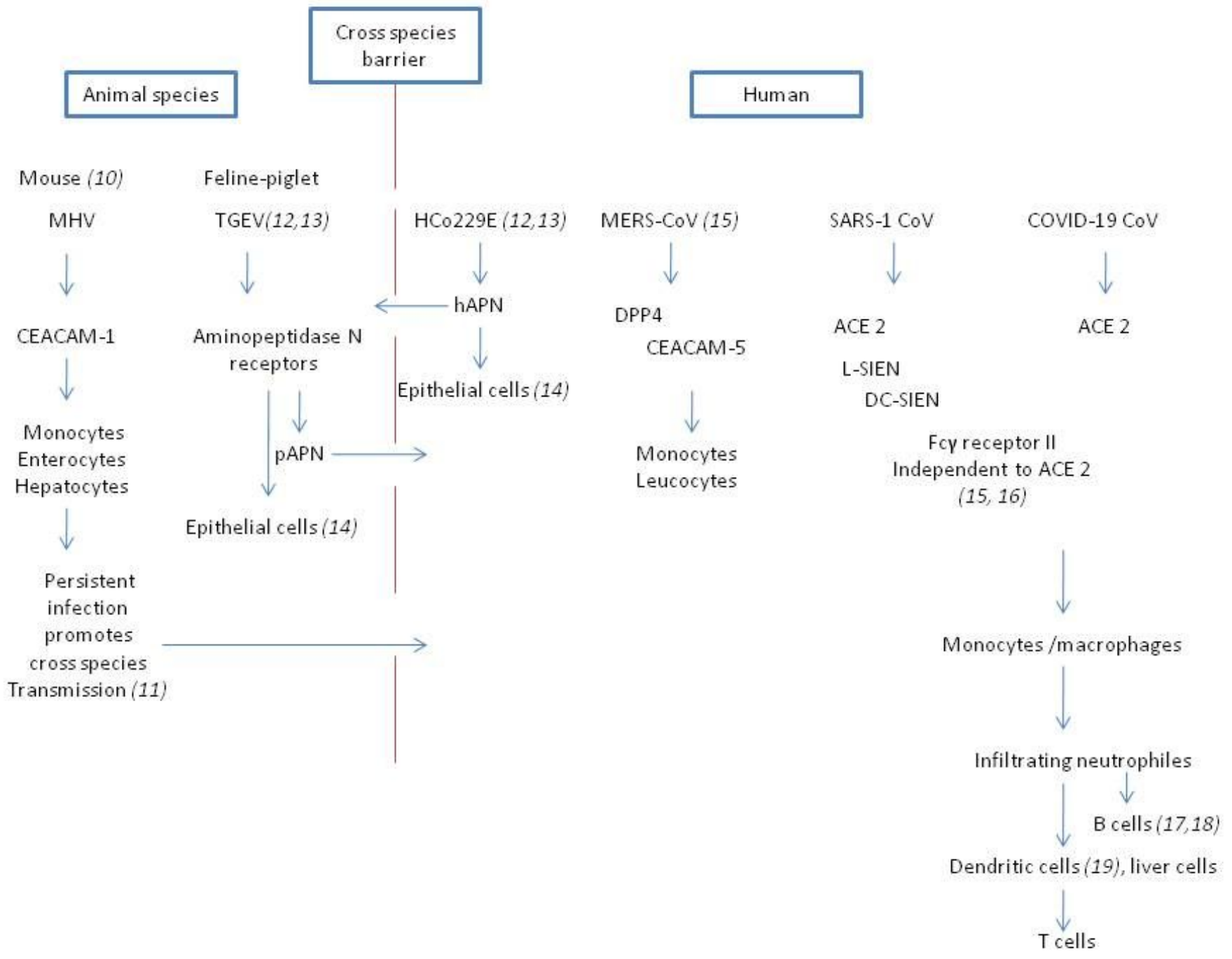

\section{Figure 1}

Cross species barrier transmission between human and animals by coronaviruses. The cross species barrier infection is achieved by coronaviruses using suitable receptors that enable this transmission. Molecules like the carcinoembryonic antigen-related adhesion molecules (CEACAM) comprise a family of antigens which are highly preserved between animal and human species and their participation can lead to a wide immune cellular dispersal of coronaviruses throughout the human organism [9]. pAPN: Piglet aminopeptidase N receptor; hAPN: Human aminopeptidase N receptor; L-SIEN: Liver/Lymph node specific intracellular adhesion molecule 3-grabbing integrin; DC-SIEN : Dendritic cell specific intracellular adhesion molecule 3-grabbing non integrin 


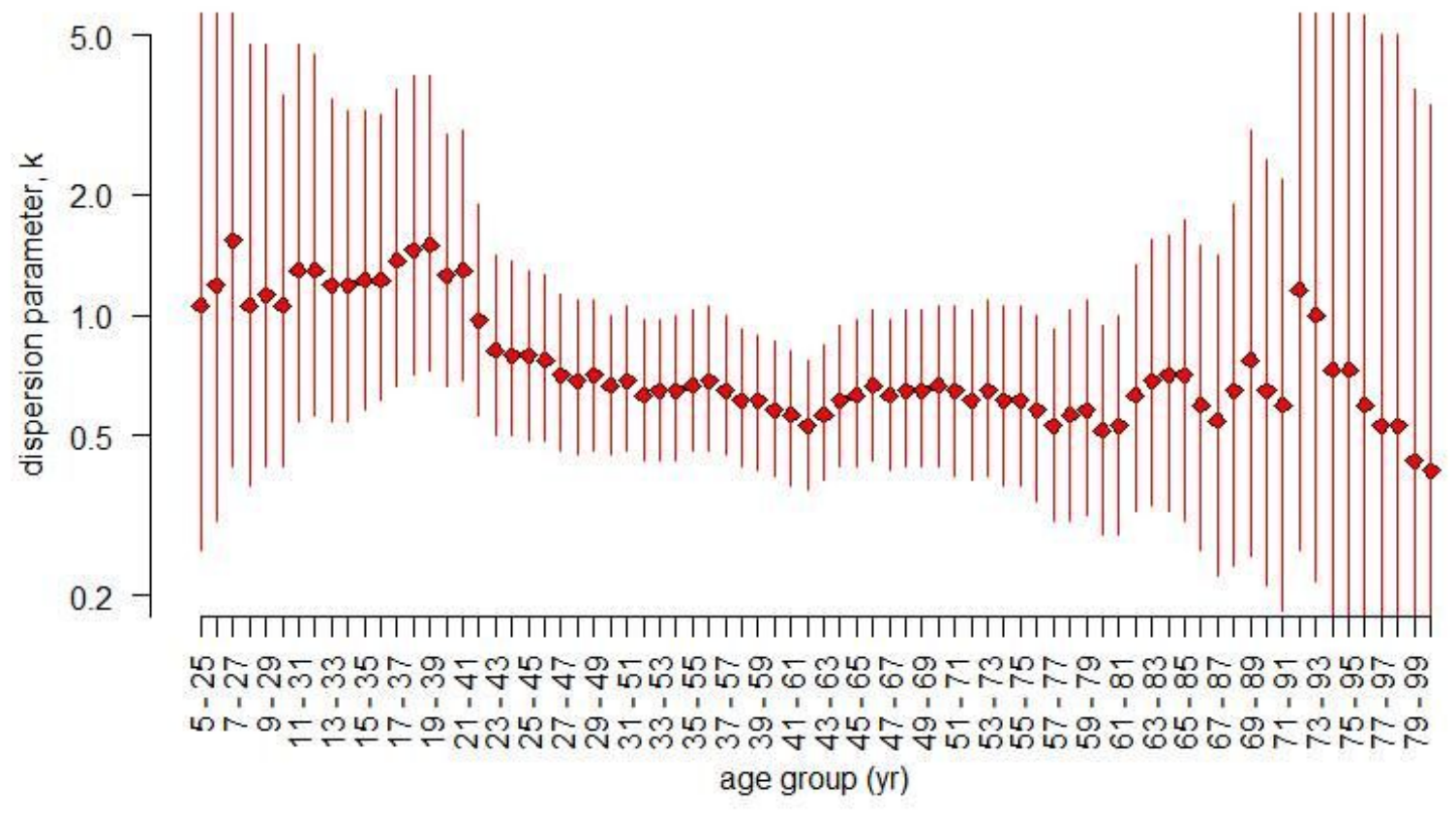

Figure 2

The dispersion parameter $(k)$. The estimated dispersion parameter $(k)$ in different age sub-groups. The dots are the estimates, and the vertical bars are the $95 \% \mathrm{Cl}$. 
The difference of dispersion parameter $(k)$ with age corresponds to the variance of individual infector's ability to transmit the disease.

Holding $\mathrm{R}$ constant e.g. $=\mathbf{2}$ for both young and older groups

Larger $K$ for young group $\rightarrow$ less

heterogeneity $\rightarrow$ difficult to control by NPIs

For four seed cases for population $<20$ years old

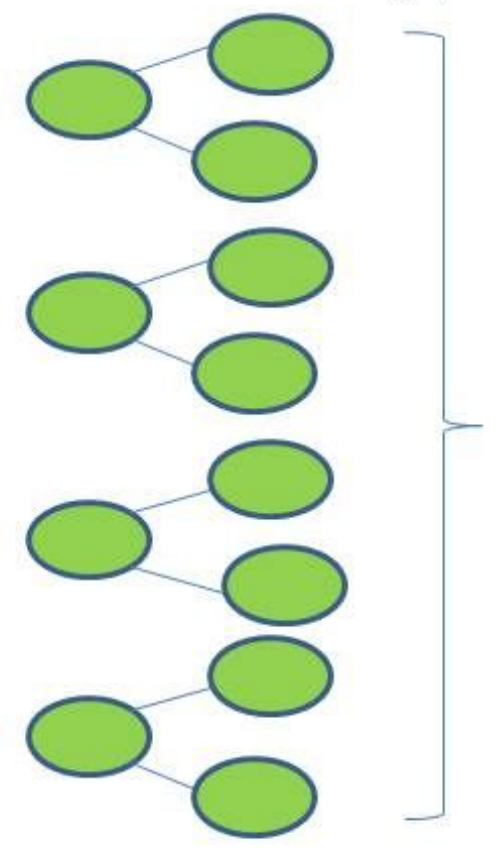

Smaller $K$ for young group $\rightarrow$ greater heterogeneity $\rightarrow$ easier to control by NPIs

For four seed cases for population $>20-60$ years old

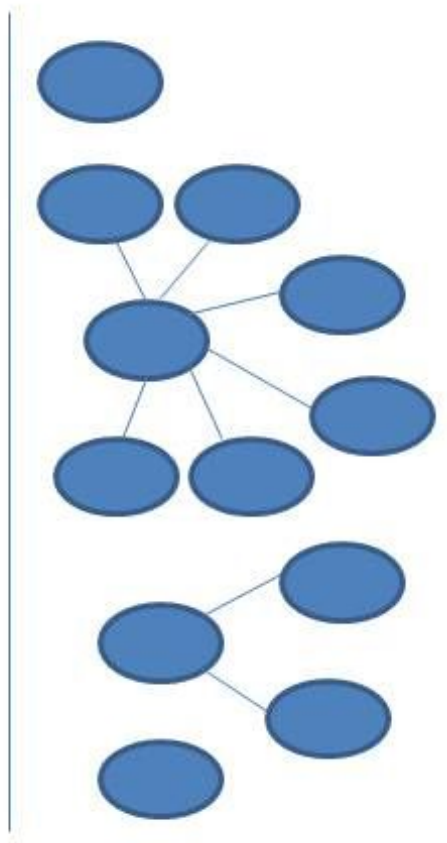

Each infector produces a different number of offsprings and most produce none

$\mathrm{R}=$ Reproduction number $K=$ dispersion parameter NPIs= Non Pharmaceutical Interventions

Figure 3

The difference of $(k)$ between age groups corresponds to the ability of infectors to transmit the disease. 